\title{
Prolongation of Anti-Inflammatory Activity of Glucocorticosteroids Encapsulated in Large Oligolamellar Liposomes in Treatment of Arthritis in Rabbits
}

\author{
O. A. Rosenberg, PhD, ScD*; A. A. Seiliev, PhD; A. Ed. Shulga; \\ A. G. Zhuikov, PhD; V. A. Volchkov, PhD, ScD \\ Russian Research Center of Radiology and Surgery Technologies, St. Petersburg, Russia
}

\begin{abstract}
Background: Liposomes have been shown to be an effective targeted drug delivery system used to decrease side effects of glucocorticosteroids in the treatment of rheumatoid arthritis.

Materials and Results: Experimental arthritis was induced in rabbits by a single intra-articular administration into the knee joint of poly-D-lysine (molecular weight, $175 \mathrm{kDa}$ ) and hyaluronic acid (7.5 $\mathrm{mg}$ per administration). To determine temperature readings over the joint a standard radiator was used with a temperature of $32^{\circ} \mathrm{C}$. Large oligolamellar liposomes from different phospholipids and and cholesterol containing hydrocortisone acetate in lipid phase and prednisolone hemisuccinate in water phase were used.

Conclusion: Intra-articular administration of the water-soluble prednisolone hemisuccinate $(0.125 \mathrm{mg})$ and the lipidsoluble hydrocortisone acetate $(0.125 \mathrm{mg})$ into the knee joint in the aqueous and lipid phases of large oligolamellar TSL (DPPC +20 mole \% cholesterol) prolongs the anti-inflammatory effect produced by glucocorticoids by 7-8 days compared to 1 day for free glucocorticosteroids at a total dose of $2.5 \mathrm{mg}$ and 2 days for phosphatidylcholine-cholesterol liposomes at a total dose of 0.25 $\mathrm{mg}$ in rabbits with aseptic arthritis. (International Journal of Biomedicine. 2017;7(2):131-134.)
\end{abstract}

Key Words: aseptic arthritis • thermosensitive liposomes • glucocorticosteroids • anti-inflammatory activity

\section{Abbreviations}

DPPC, dipalmitoylphosphatidylcholine; HA, hydrocortisone acetate; PH, prednisolone hemisuccinate; PhC, phosphatidylcholine TSL, thermosensitive liposomes.

\section{Introduction}

Since common treatments for rheumatoid arthritissuch as nonsteroidal anti-inflammatory drugs, corticosteroids, disease modifying anti-rheumatic drugs and some biological agents-have proven to be unable to achieve drug-free remission, ${ }^{(1)}$ a number of targeted drug delivery strategies have been developed in order to attenuate side effects to other tissues. These include microemulsions, microspheres,

*Corresponding author: Prof. Oleg Rosenberg, PhD, ScD Department of Medical Biotechnology of Russian Research Center of Radiology and Surgery Technologies, St. Petersburg, Russia. E-mail: rozenberg@biosurf.ru liposomes and others, of which liposomes have been shown to retain the drug in the synovial cavity effectively due to their chemical composition and size. ${ }^{(2,3)}$ In our previous work we have demonstrated prolongation of the anti-inflammatory effect produced by HA encapsulated into the membrane of multilamellar TSL composed of DPPC and cholesterol in rabbits with aseptic arthritis. ${ }^{(4)}$ However, this prolongation was limited to only about 5 days compared to 1 day in the case of intra-articular administration of free hydrocortisone acetate or 2 days for its liposomal form composed of egg lecithin and cholesterol. At the same time, no attempts have been done so far to study the effectiveness of administration of a combination of water-soluble and lipid-soluble glucocorticosteroids entrapped in the aqueous and lipid phases of 1 liposome formulation. 
The study was aimed at evaluating the potential for prolonging the anti-inflammatory activity of glucocorticosteroids encapsulated into TSL composed of DPPC and cholesterol, with the aqueous phase containing water-soluble $\mathrm{PH}$ and the lipid phase containing lipid soluble HA.

We compared glucocorticosteroids entrapped in liposomes with free glucocorticosteroids at a 10 times higher dose. Two types of liposomes were compared - eggphosphatidylcholine $(\mathrm{PhC})$ liposomes with a melting phase transition temperature $\left(\mathrm{T}_{\mathrm{m}}\right)$ of $+10^{\circ} \mathrm{C}$ and DPPC liposomes with a $\mathrm{T}_{\mathrm{m}}$ of $41.5^{\circ} \mathrm{C}$. Adding cholesterol to the liposome membrane decreases the amplitude and increases the $\mathrm{T}_{\mathrm{m}}$ range. If liposomes are made from DPPC and 20\% mole cholesterol, their membrane is in a metastable state over the temperature range from $37^{\circ} \mathrm{C}$ to $47^{\circ} \mathrm{C}$, including the inflammation temperature range. ${ }^{(5)}$

\section{Methods}

Experimental arthritis was induced in rabbits by a single intra-articular administration into the knee joint of poly-Dlysine (molecular weight $-175 \mathrm{kDa}$ ) and hyaluronic acid (7.5 $\mathrm{mg}$ per administration) ${ }^{(6)}$ There were four groups of rabbits containing 5 animals each. The first group received $0.5 \mathrm{ml}$ saline, the second group received a $0.5 \mathrm{ml}$ mixture of $\mathrm{PH}(1.25$ $\mathrm{mg})$ and HA $(1.25 \mathrm{mg})$, the third group and the fourth received liposomal form containing both glucocorticosteroids at a 10 times lower dose $(0.125 \mathrm{mg})$, the third group receiving egg$\mathrm{PhC}$ liposomes and cholesterol and the fourth group receiving DPPC liposomes and cholesterol. In all groups, the injection was made on the third day after arthritis induction and at the very peak of the inflammatory reaction. Large oligolamellar liposomes were obtained using the reverse-phase technique.(7) Egg-PhC and DPPC purchased from Lipoid, Germany, and cholesterol purchased from Avanti Polar Lipids, Inc., USA, were taken at a ratio of 7:2 (molar), $21 \mu \mathrm{mol}$ and $6 \mu \mathrm{mol}$ correspondingly, and placed into a round-bottom flask. Then $\mathrm{HA}$ in chloroform and $1 \mu \mathrm{Curie}$ of ${ }^{3} \mathrm{H}-\mathrm{HA}$ (specific activity of $48 \mu \mathrm{Curie} / \mu \mathrm{mol}$, Izotop, St Petersburg, Russia) were added. The solvent was removed by a rotary evaporator to achieve dryness; the lipid film was cleared of residual chloroform under vacuum by an evaporator (Rotavapor R-114, Buche, Switzerland), dissolved in $3 \mathrm{ml}$ of freshly prepared diethyl ether and $9 \mathrm{ml}$ of saline containing $5 \mathrm{mg}$ of $\mathrm{PH}$ and $1 \mu \mathrm{Cu}$ of ${ }^{51} \mathrm{Cr}$-EDTA (specific activity $100 \mu \mathrm{Cu} / \mathrm{ml}$, Izotop,Russia). The system was processed in a sonicator (UZD-H-1, Sumy, Ukraine) at a frequency of $22 \mathrm{kHz}$ and a power $630 \mathrm{~W}$ of at $+4^{\circ} \mathrm{C}$ under argon protection. The obtained reverse emulsion (oil-in-water) was transformed into regular emulsion (waterin-oil) by eliminating the organic solvent using a rotary evaporator (Rotavapor R-114, Buche, Switzerland). Then the liposome emulsion was kept for two hours at $37^{\circ} \mathrm{C}$ for egg-PhC and at $50^{\circ} \mathrm{C}$ for DPPC and centrifuged for 1 hour at $10000 \times \mathrm{g}$ using a Sigma 6K10 centrifuge, Germany, to spin down liposomes. Preliminary experiments have shown HA encapsulation into the liposome lipid phase to be $96 \%-98 \%$ as calculated from ${ }^{3} \mathrm{H}-\mathrm{HA}$ inclusion into the liposome membrane, while PH encapsulation has been shown to amount to $30 \%$
$36 \%$ as calculated from ${ }^{51} \mathrm{Cr}$-EDTA inclusion. The liposome residues were resuspended in the saline to reach $\mathrm{HA}$ and $\mathrm{PH}$ concentrations of $0.25 \mathrm{mg} / \mathrm{ml}$ each. The lipid concentration in samples amounted to $2 \mathrm{mg} / \mathrm{ml}$. The liposome samples were used within 1 week. Liposomal preparations were checked for sterility, and whenever possible argon protection was used. The inflammatory reaction in the joint was registered by a thermal camera. To determine temperature readings over the joint a standard radiator (Pergamed, Russia) was used with a temperature of $32^{\circ} \mathrm{C}$. Both hyperthermia severity and hyperthermic area, calculated from negative images using planimetric analysis, were taken into account. Experiment was performed in accordance with the Guide for the Care and Use of Laboratory Animals (Institute of Laboratory Animal Resources, 1996)

Statistical analysis was performed using the statistical software «Statistica» (v6.0, StatSoft, USA). The mean (M) and standard error of the mean (SEM) were calculated. The Wilcoxon criterion was used to compare the differences between the paired samples. Pearson's correlation coefficient (r) was used to determine the strength of the relationship between the two continuous variables. A probability value of $\mathrm{P}<0.05$ was considered statistically significant.

\section{Results}

A mixture of free HA and PH at a dose of $2.5 \mathrm{mg}(1.25 \mathrm{mg}$ per each glucocorticosteroid) caused a statistically significant $(\mathrm{P}<0.001)$ decrease in temperature over the joint 24 hours after the administration, which came back to baseline 1 day after the decrease (i.e. the anti-inflammatory effect lasted for a little over 1 day) (Fig.1). Glucocorticosteroids encapsulated in the aqueous and lipid phases of PhC/cholesterol liposomes $(0.125 \mathrm{mg}$ of each glucocorticosteroid amounting to 0.25 $\mathrm{mg}$ ) also had an effect 1 day after administration (Fig.1), but their effect was statistically significantly $(\mathrm{P}<0.01)$ prolonged up to 2 days, the total dose being 10 times less than in free glucocorticosteroids.

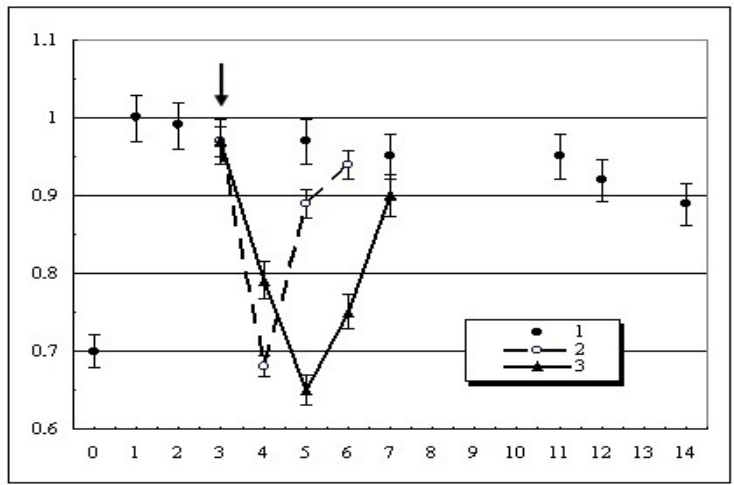

Fig. 1. Anti-inflammatory effect of mixed water-soluble and lipid-soluble free or encapsulated into lecithin-cholestrol liposomes glucocorticoids.

$\mathrm{X}$-line: time after intra-articular administration of poly-D-lysine and hyaluronic acid (days),

Y-line: relative thermometrical index (relative units). The arrow indicates the time of drug administration.

(1) - no treatment; (2) - administration of mixed HA and PH (1.25 mg of each drug amounting to a total of $2.5 \mathrm{mg}$ ); (3) liposomes from $\mathrm{PhC}$ and $20 \%$ mole cholesterol containing $0.125 \mathrm{mg}$ of $\mathrm{HA}$ and $0.125 \mathrm{mg}$ of $\mathrm{PH}$ ( $0.5 \mathrm{mg}$ of lipids in $0.25 \mathrm{ml}$ of saline). 
The anti-inflammatory effect calculated from both hyperthermia severity and inflammation area yields consistent results (Fig.2). The correlation test showed a strong positive correlation between these parameters: $\mathrm{r}=+0.88 \pm 0.180(\mathrm{P}<0.01)$. The structure and permeability of liposomes are known to change in the lipid melting phase-transition temperature $\left(\mathrm{T}_{\mathrm{m}}\right)$ range.$^{(8)}$ This property of lipids is used to increase the rate of drug release from the aqueous phase of the vesicles due to hyperthermia. There are a number of reasons to believe that bringing closer the phase states of the vesicle membrane and the plasma membrane of the target cells, which are synovial cells in this case, may facilitate their interaction. ${ }^{(9)}$

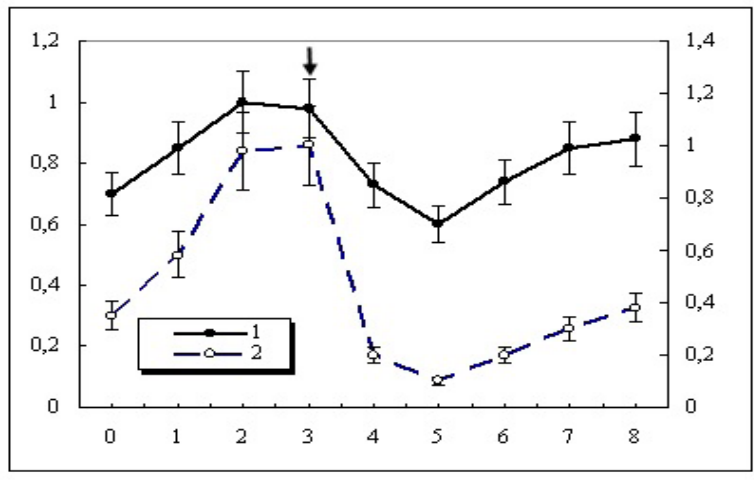

Fig. 2. Hyperthermia severity and hyperthermic area correlation.

X-line - time after arthritis induction (days). Y-line (left) - thermometric index; (right) - inflammation area index (relative units).

(1) - thermometric index after administration of mixed water-soluble and lipid-soluble encapsulated into egg-PhC and $20 \%$ mole cholesterol liposomes glucocorticoids; (2) - inflammation area index.

The duration of the anti-inflammatory effect produced by mixed glucocorticosteroids (HA and $\mathrm{PH}$ ) entrapped in DPPC/cholesterol liposomes proved to be significantly longer, reaching 7-8 days after the drop in temperature (Fig.3).

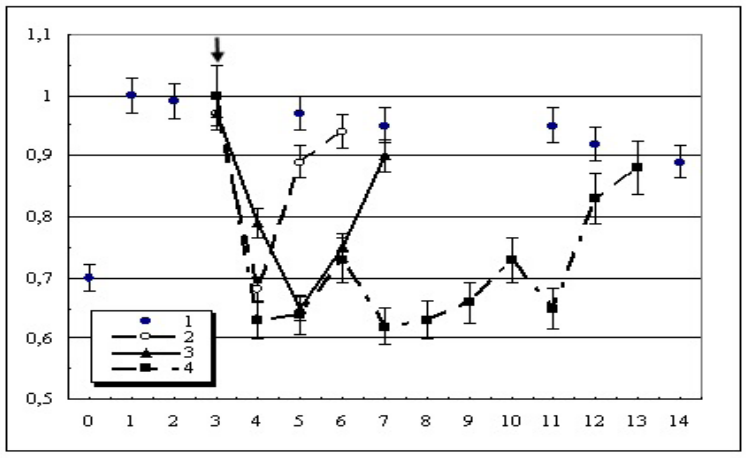

Fig. 3. Prolongation of the anti-inflammatory effect of $\mathrm{HA}$ and $\mathrm{PH}$ encapsulated in the liposomal membrane with various melting phase transition temperature.

X-line: time after intra-articular administration of poly-D-lysine and hyaluronic acid (days),

Y-line: relative thermometrical index (relative units). The arrow indicates the time of drug administration.

(1) - no treatment; (2) - administration of mixed HA and PH (1.25 mg of each drug amounting to a total of $2.5 \mathrm{mg}$ ); (3) - liposomes from $\mathrm{PhC}$ and $20 \%$ mole cholesterol containing $0.125 \mathrm{mg}$ of $\mathrm{HA}$ and $0.125 \mathrm{mg}$ of $\mathrm{PH}$ (0.5 mg of lipids in $0.25 \mathrm{ml}$ of saline); (4) - liposomes from DPPC and $20 \%$ mole cholesterol containing $0.125 \mathrm{mg}$ of $\mathrm{HA}$ and $0.125 \mathrm{mg}$ of $\mathrm{PH}$ ( $0.5 \mathrm{mg}$ of lipids in $0.25 \mathrm{ml}$ of saline).
The temperature drop rate over the joint was the same for the both types of liposomes. However, when DPPC/ cholesterol liposomes were used, the anti-inflammatory effect lasted for up to 7-8 days after administration.

\section{Discussion}

This shows that the prolonged effect of the antiinflammatory action produced by mixed glucocorticosteroids (HA and PH) entrapped in DPPC/cholesterol liposomes is due to both HA present in the lipid phase and $\mathrm{PH}$ present in the aqueous phase of the liposomes. Since HA alone encapsulated in DPPC/cholesterol liposomes yielded a prolongation of only 5 days, ${ }^{(4)}$ the greater prolongation effect can be attributed only to the fact that water soluble $\mathrm{PH}$ was also administered. It can also be assumed that when DPPC/cholesterol liposomes come into contact with inflammatory cells within the joint, the effectiveness of inflammatory cell (neutrophils, monocytes) membrane merger depends on the closeness of the phase state of liposome lipids and the inflammatory cells at inflammation temperature. Due to the hypothermic effect of the mixed glucocorticosteroids and the temperature decrease down to $32^{\circ} \mathrm{C}$, the vesicles in the joint change to the solid crystalline state so that the period of their utilization increases as they stay there as a depot. However, in this state the water-soluble $\mathrm{PH}$ leaves the liposomes along a concentration gradient, which leads to an additional anti-inflammatory effect. The temperature rise peak during the third day after the first temperature drop following the administration of DPPC/cholesterol liposomes is of special importance. We believe that a slight increase of temperature followed by a temperature decrease over a short period of time indicates that the temperature increase in the joints transforms DPPC/cholesterol liposomes once again from the solid crystalline state into liquid crystalline state and HA once again shows an anti-inflammatory effect. This phenomenon clearly demonstrates that the activity of HA encapsulated in DPPC/cholesterol liposomes is temperature related.

\section{Competing interests}

The authors declare that they have no competing interests.

\section{Acknowledgements}

We thank Evgenia Zvonareva for technical assistance and translation of the manuscript into English.

\section{References}

1. Massarotti EM. Clinical and patient-reported outcomes in clinical trials of abatacept in the treatment of rheumatoid arthritis. Clin Ther. 2008;30(3):429-42. doi: 10.1016/j. clinthera.2008.03.002.

2. Kapoor B, Singh SK, Gulati M, Gupta R, Vaidya Y. Application of liposomes in treatment of rheumatoid arthritis: quo vadis. Scientific World Journal. 2014;2014:978351. doi: $10.1155 / 2014 / 978351$. 
3. Curtis JR, Singh JA. Use of biologics in rheumatoid arthritis: current and emerging paradigms of care. Clin Ther. 2011;33(6):679-707. doi: 10.1016/j.clinthera.2011.05.044.

4. Davidenkova EF, Ternova NK, Rozenberg OA, Noskin LA, Loshakova LV. [Relation between the prolongation of the anti-inflammatory activity of liposome-encapsulated hydrocortisone and liposome composition in experimental arthritis]. Biull Eksp Biol Med. 1984;97(6):656-8. [Article in Russian].

5. Ivkov VG, Berestovsky GN. Dynamic structure of the lipid bilayer. Moscow: Nauka; 1981.

6. Dingl JT, Gordon JL, Hazelman BL, Knight CG, Page Thomas DP, Phillips NC, et al. Novel treatment for joint inflammation. Nature. 1978; 271(5643):372-3.

7. Szoka F Jr, Papahadjopoulos D. Procedure for preparation of liposomes with large internal aqueous space and high capture by reverse-phase evaporation. Proc Natl Acad Sci USA. 1978; 75(9):4194-8.

8. Antonov VF, Anosov AA, Norik VP, Korepanova EA, Smirnova EY. Electrical capacitance of lipid bilayer membranes of hydrogenated egg lecithin at the temperature phase transition. Eur Biophys J. 2003 Mar;32(1):55-9.

9. Rozenberg OA, Bekreneva VIu, Loshakova LV, Rezvaia SP, Davidenkova EF. [Specificity of liposome uptake from target cell lipids]. Biull Eksp Biol Med. 1984;97(6):670-2. [Article in Russian] 